\title{
A Scattering-Type Transverse Resonance Technique for the Calculation of (M) MIC Transmission Line Characteristics
}

\author{
Jens Bornemann, Senior Member, IEEE
}

\begin{abstract}
A scattering-type formulation of the transverse resonance technique is introduced and applied to a variety of currently practical (M)MIC configurations. By utilizing a reflection coefficient matrix representation of boundary conditions, the characteristics of open, conductor-backed and shielded microstrip, slotline, or coplanar waveguide can be calculated. Excellent agreement with measurements and theoretical data on fundamental and higher-order mode characteristics is obtained. In contrast to other methods, which require mainframe support, the software based on this formulation is operational on 386compatible personal computers.
\end{abstract}

\section{INTRODUCTION}

$\mathrm{T}$ HE CONVENTIONAL transverse resonance method for transversely discontinuous waveguides [1] such as finlines or shielded microstrip is a well-known technique to calculate the propagation characteristics of quasi-planar transmission lines structures. Although the restrictions to moderate and larger slotwidths [2], [3] can be reduced by using an improved transmission-matrix formulation for cascaded discontinuities [4], precisely defined boundary conditions are still required to formulate the resonance condition of a structure. Whereas many shielded configurations with electric-wall boundaries have been analyzed for their propagation constants and characteristic impedances, open structures as applied in (M)MIC circuitry have not yet been solved with this model. An admittance approach of the transverse resonance technique has been used to investigate partially open configurations such as the groove guide [5] and the dielectric image guide [22]. However, since open and short-circuit boundaries are introduced in terms of tangent and cotangent functions [22], respectively, singularities for certain combinations of dimensions and frequency constitute a potential source for numerical inaccuracies. These problems are unknown to the scattering-type formulation presented here.

Modern integrated circuit technologies, such as MIC, MHMIC and MMIC, employ conductor backing and shielding, which influence and, in most cases, invalidate circuit parameter values computed by conventional methods. Problems have been addressed which are related to

Manuscript received March 28, 1991; revised July 12, 1991.

The author is with the Laboratory for Lightwave Electronics, Microwaves and Communications, Department of Electrical and Computer Engineering, University of Victoria, Victoria, BC, Canada.

IEEE Log Number 9102781 conductor-backing effects [6], [7], mode conversion [8], dense packaging [9], [10], leakage [11] and radiation [12]. Most of the theoretical models available, however, are presented for very specific problems, thereby neglecting important parameters such as the strip thickness, e.g., [11], [12], are based on static approaches [13], [14], which become invalid even for medium-low frequencies, or rely heavily on mainframe computer support [15]-[17]. Another method, which is based on modifications of simplified models, is demonstrated for microstrip-like structures only [18]. None of these methods combine a generality in analyzable structures with workstation-applicable software.

Therefore, a scattering-type formulation of the transverse resonance technique is introduced in this paper. This method allows the calculation of different (M)MIC transmission line structures, such as microstrip, slotline and coplanar waveguide, in open, conductor-backed or shielded circuitries Fig. 1 by using one and the same algorithm. By introducing transverse boundary conditions in terms of reflection coefficient matrices, the transverse resonance procedure can easily be adapted to include open, conductor-backed and shielded configurations while the resonance condition itself remains unchanged. Moreover, the method preserves numerical stability by allowing the number of expansion terms in different subregions to be individually selected. The main advantages of this formulation are, firstly, that the Fortran software code is operational on 386-type personal computers, while competitive models usually require main-frame support and, secondly, that although losses have not been included, the results in terms of effective permittivity and characteristic impedance are in good agreement with those obtained by the corresponding competitive models.

\section{THEORY}

According to the scattering-type formulation of the transverse resonance method, the electro-magnetic field in subregions $i=0$-IV (c.f., Fig. 2(a)):

$$
\begin{aligned}
& \vec{E}^{i}=\frac{1}{j \omega \epsilon^{i}} \nabla \times \nabla \times\left(A_{e x}^{i} \vec{e}_{x}\right)+\nabla \times\left(A_{h x}^{i} \vec{e}_{x}\right) \\
& \vec{H}^{i}=\frac{-1}{j \omega \mu} \nabla \times \nabla \times\left(A_{h x}^{i} \vec{e}_{x}\right)+\nabla \times\left(A_{e x}^{i} \vec{e}_{x}\right)
\end{aligned}
$$


(a)

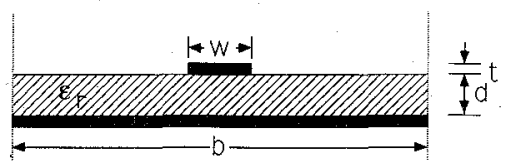

(b)

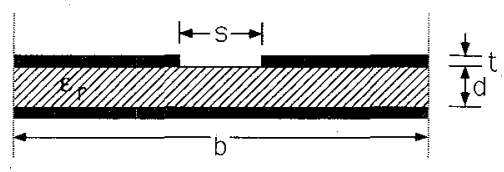

(c)

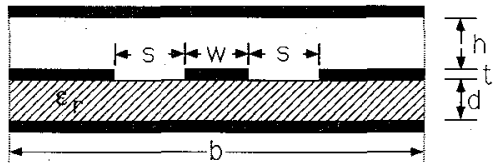

Fig. 1. Some examples of (M)MIC structures. (a) Open microstrip line. (b) Conductor-backed slotline. (c) Shielded coplanar waveguide.

is characterized by incident and reflected waves in $x$ directions. Therefore, in regions $i=0, \mathrm{I}, \mathrm{II}, \mathrm{IV}$, the potential functions read

$$
\begin{aligned}
A_{h_{x}}^{i}= & \sum_{n=1}^{N}\left(A_{n}^{i} e^{-j k_{x n}^{i} x}+B_{n}^{i} e^{+j k_{x n}^{i} x}\right) \\
& \cdot \cos \left\{(2 n-1) \frac{\pi}{b} y\right\} e^{-j k_{z} z} \\
A_{e_{x}}^{i}= & \sum_{n=1}^{N} \frac{1}{j k_{x n}^{i}}\left(-C_{n}^{i} e^{-j k_{x n}^{i} x}+D_{n}^{i} e^{+j k_{x n}^{i} x}\right) \\
& \cdot \sin \left\{(2 n-1) \frac{\pi}{b}\right\} e^{-j k_{z} z}
\end{aligned}
$$

and in region III:

$$
\begin{aligned}
A_{h x}^{I I I}= & \sum_{m=0}^{M-1}\left(A_{m}^{I I I} e^{-j k_{x m}^{I I I} x}+B_{m}^{I I I} e^{+j k_{x m}^{I I I} x}\right) \\
& \cdot \cos \left\{\frac{m \pi}{s}(y-c)\right\} e^{-j k_{z} z} / \sqrt{1+\delta_{o m}} \\
A_{e x}^{I I I}= & \sum_{m=1}^{M-1} \frac{1}{j k_{x m}^{I I I}}\left(-C_{m}^{I I I} e^{-j k_{x m}^{I I I} x}+D_{m}^{I I I} e^{+j k_{x m}^{I I I} x}\right) \\
& \cdot \sin \left\{\frac{m \pi}{s}(y-c)\right\} e^{-j k_{z} z} .
\end{aligned}
$$

$A^{i}, B^{i}$ and $C^{i}, D^{i}(i=0-\mathrm{IV})$ are amplitude coefficients, which are combined to vectors $\underline{F}^{i}$ and $\underline{R}^{i}$, respectively, in Fig. 2(a), and $\delta_{o m}$ in (5) is the Kronecker delta.

Due to the flexible slot position and size in region III, and to the different conditions at $y=0$ and $y=b / 2$ (electric and magnetic wall, respectively, c.f., Fig. 2(a)), this method offers the possibility of analyzing all (M)MIC structures which are currently of practical interest. The microstrip structure of width $w$ is calculated by letting $c=0$, hence $s=b / 2-w / 2$. Furthermore, with $c=0$ but small $s$, a slotline of slotwidth $2 \mathrm{~s}$ is formed. The general structure for the coplanar waveguide is shown in Fig. 2(a). It should also be noted that, in general, coupled slots can be computed in terms of their even and odd mode characteristics. The main advantage of this method is that the

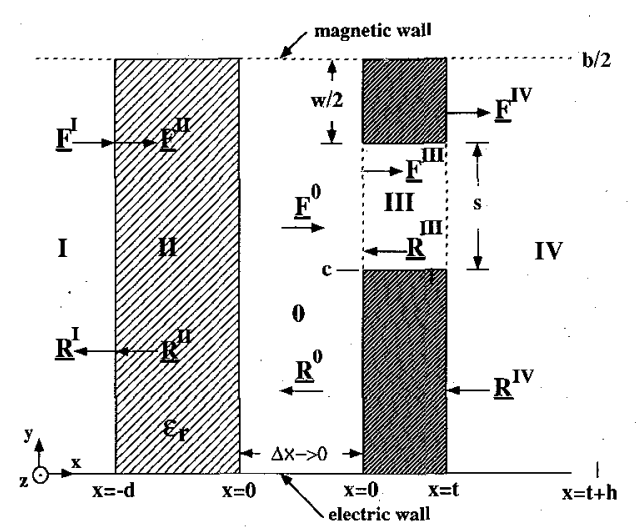

(a)

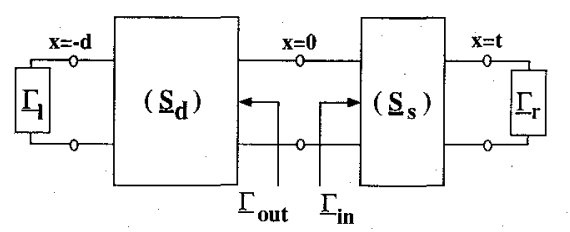

(b)

Fig. 2. Field theory treatment. (a) Subregions and wave amplitude vectors. (b) Scattering-type representation.

conditions for open, conductor-backed and shielded configurations apply to all of the above-mentioned possibilities. This is a direct consequence of the scattering-type representation of the transverse resonance method, which can briefly be described as follows: By matching the tangential field components at interfaces $x=-d, 0$ and $x=0, t$ the modal scattering-type representation of the dielectric substrate $\left(\underline{S}_{d}\right)$ and the metallization $\left(\underline{S}_{s}\right)$ are obtained (c.f., Fig. 2(b)). The only difference to the generalized scattering matrix is that the amplitude coefficients are not normalized and, therefore, may exceed the unity value. As $\Delta x$ approaches zero (Fig. 2(a)), vectors $\underline{F}^{0}$ and $\underline{R}^{0}$ are related by

$$
\underline{R}^{0}=\underline{\Gamma}_{\mathrm{in}} \underline{F}^{0} \text { and } \underline{F}^{0}=\underline{\Gamma}_{\text {out }} \underline{R}^{0}
$$

(c.f., Fig. 2(b)). Hence the transverse resonance condition can be formulated as

$$
\left[\underline{I}-\underline{\Gamma}_{\mathrm{in}} \underline{\mathrm{O}}_{\mathrm{out}}\right] \underline{R}^{0}=0
$$

where $\underline{I}$ represents the identity matrix. For the different cases of open, conductor-backed or shielded configurations, the reflection coefficient matrices $\underline{\Gamma}_{\text {in }}, \underline{\Gamma}_{\text {out }}$ are given by

\section{Open structure:}

$$
\underline{\Gamma}_{l}=\underline{\Gamma}_{r}=0, \underline{\Gamma}_{\mathrm{in}}=\underline{S}_{S 11}, \underline{\Gamma}_{\text {out }}=\underline{S}_{d 22} ;
$$

Conductor-backed structure:

$$
\underline{\Gamma}_{r}=0, \underline{\Gamma}_{l}=-1, \underline{\Gamma}_{\text {out }}=\underline{S}_{d 22}-\underline{S}_{d 21}\left[\underline{I}+\underline{S}_{d 11}\right]^{-1} \underline{S}_{d 12}
$$

with $\Gamma_{\text {in }}$ as in (9);

Shielded structure:

$$
\left(\underline{\Gamma}_{r}\right)=\left(\begin{array}{cc}
\underline{D} & \underline{0} \\
\underline{0} & \underline{D}
\end{array}\right)
$$




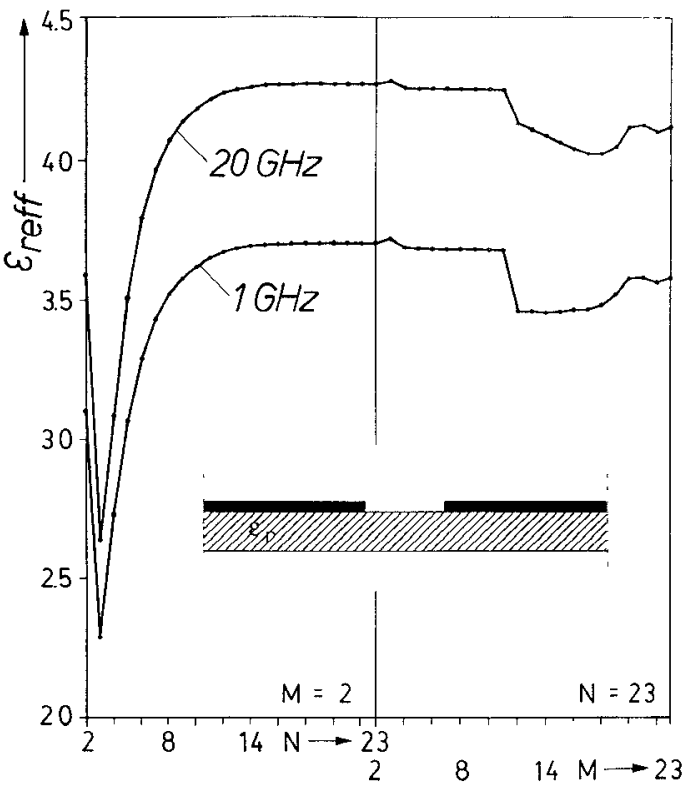

Fig. 3. Convergence analysis for open slotline; $N=$ number of expansion terms in regions 0 , I, II, IV; $M=$ number of terms in region III.

where

$$
\begin{gathered}
\underline{D}=\operatorname{diag}\left\{-e^{-\jmath 2 k_{x n}^{I V} h}\right\}, \\
\underline{\Gamma}_{\mathrm{in}}=\underline{S}_{S 11}+\underline{S}_{S 12} \underline{\Gamma}_{r}\left[\underline{I}-\underline{S}_{S 22} \underline{\Gamma}_{r}\right]^{-1} \underline{S}_{S 21},
\end{gathered}
$$

and $\underline{\Gamma}_{l}, \underline{\Gamma}_{\text {out }}$ as in (10).

The zeros of the determinant in (8) specify the effective permittivity values. After solving for the amplitude vectors $\underline{R}^{i}$ and $\underline{F}^{i}$, the characteristic impedance is calculated using the power-voltage definition.

The convergence behavior of this method is demonstrated in Fig. 3 at the example of an open slotline. By keeping the number $M$ of slot terms low, a good convergence characteristic is obtained with about $N=20$ modes. Increasing the number of slot terms, however, can lead to numerical instabilities as can be seen in the right half of Fig. 3 for $M>10$. Based on this analysis, the results presented in this paper have been calculated with $N$ between 20 and 30 . For given $N, M$ is determined by the ratio of dimensions, e.g., $M=2 N s / b$. The CPU time required to calculate $\epsilon_{\text {reff }}$ and $Z_{o}$ varies between five and twenty minutes per frequency sample on a 386 compatible work station.

\section{RESULTS}

For open structures, Fig. 4 shows a comparison between data obtained by this method with theoretical and experimental results available from literature. Fig. 4(a) compares the effective permittivity with measurements carried out in [19]. Although the finite strip width of the open slotline in [19] has been replaced by the magnetic wall model in this theory, good agreement is obtained. The two slotline models (finite strip and magnetic wall) have also been analyzed in [16] where the finite strip

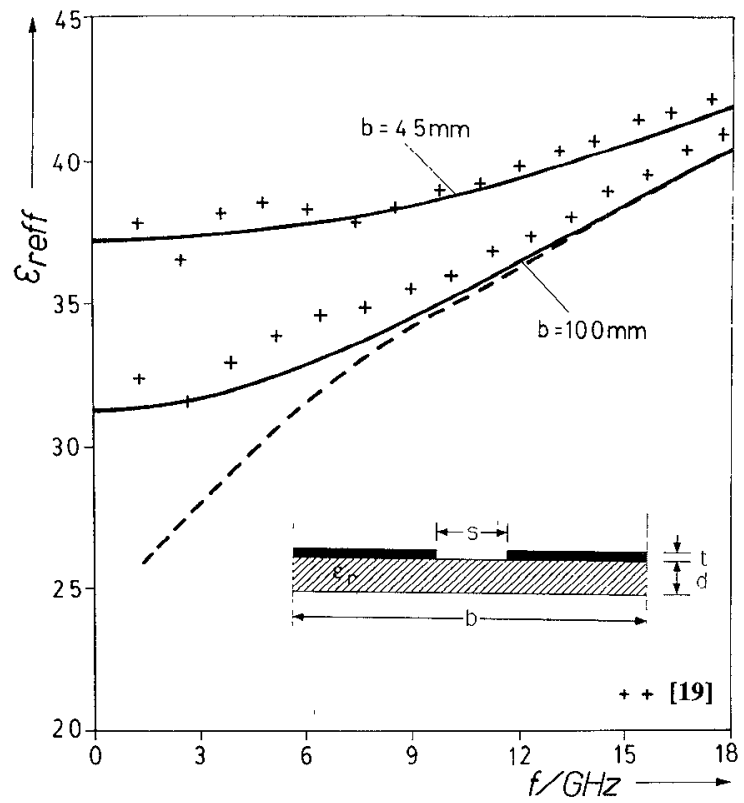

(a)

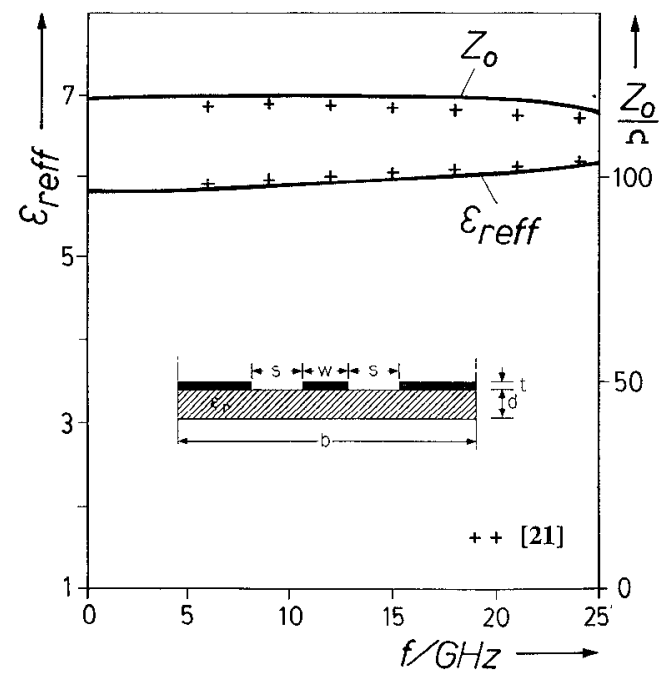

(b)

Fig. 4. Comparison of characteristics of open structures obtained using this method (solid lines) with measurements and with other theoretical data. (a) Open slotline $(++$ measurement [19], -- ridge waveguide model [20], $d=635 \mu \mathrm{m}, t=7.03 \mu \mathrm{m}, s=500 \mu \mathrm{m}, \epsilon_{r}=9.7$ ). (b) Open coplanar waveguide $(++$ theory $[21], b=4 \mathrm{~mm}, d=1 \mathrm{~mm}, t=5 \mu \mathrm{m}$, $w=300 \mu \mathrm{m}, s=250 \mu \mathrm{m}, \epsilon_{r}=11$ ).

model values are found to be slightly higher than those resulting from the magnetic wall assumption. This tendency is confirmed in Fig. 4(a) when comparing the measured values $(+)$ with the solid lines of this theory. The dashed line represents the results of Cohn's theory [20] for the open slotline. Since a ridge waveguide model is used in [20], the method fails at lower frequencies. Close agreement is also obtained by comparing the results of this method with those for the open coplanar line presented in [21] (Fig. 4(b)). The slight deviations in effective permittivity and characteristic impedance are due to the finite metallization thickness considered in the present method, as has already been observed in [3]. 


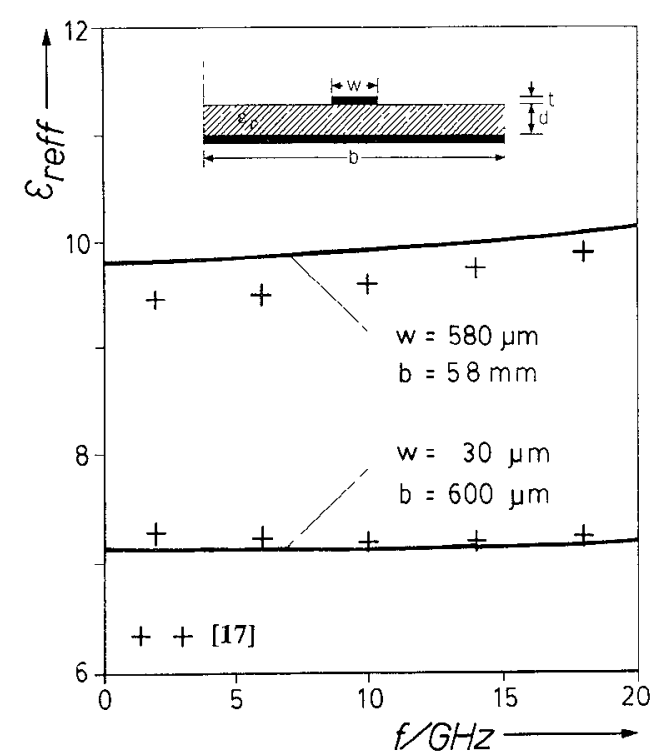

(a)

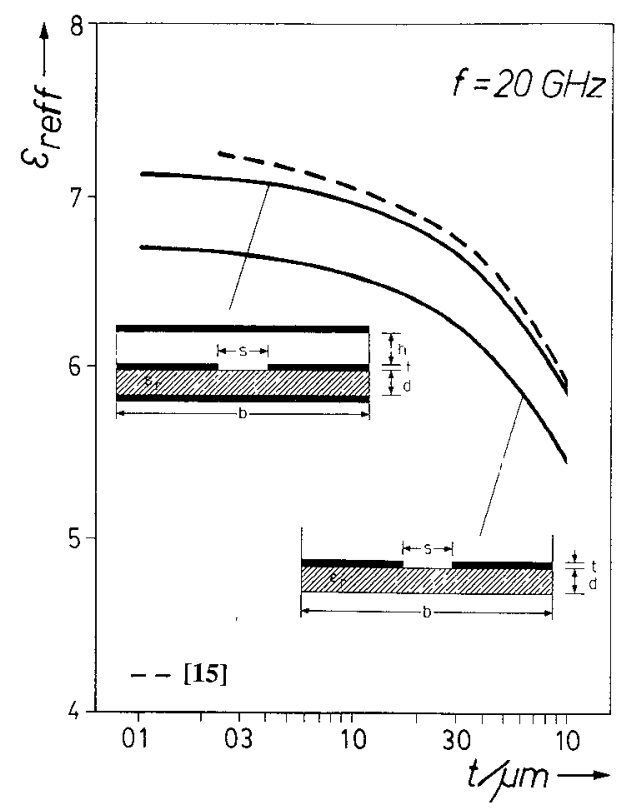

(b)

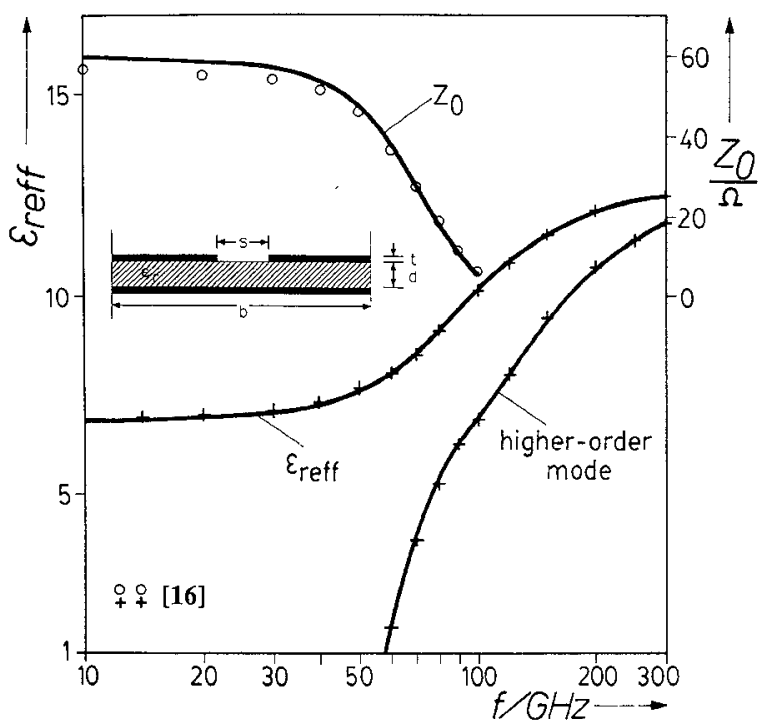

(c)

Fig. 5. Comparison between MMIC results obtained with this method (solid lines) and those of Heinrich [15]-[17]. (a) Open microstrip line $\left(++[17], d=200 \mu \mathrm{m}, t=6 \mu \mathrm{m}, \epsilon_{r}=12.9\right)$. (b) Open and shielded slotline $(--[15], b=315 \mu \mathrm{m}$, $\left.d=150 \mu \mathrm{m}, s=15 \mu \mathrm{m}, h=6 \mathrm{~mm}, \epsilon_{r}=12.9\right)$. (c) Conductor-backed slotline ( $++\circ 0$ [16], $b=840 \mu \mathrm{m}, d=600 \mu \mathrm{m}, s=40$ $\mu \mathrm{m}, t=3 \mu \mathrm{m}, \epsilon_{r}=12.9$ ).

In Fig. 5, the scattering-type transverse resonance technique is compared with Heinrich's method [15]-[17], which includes conductor losses. The lower curve in Fig. 5(a) for the open microstrip line and the upper curve in Fig. 5(b) for a shielded slotline confirm the thesis in [17] that calculations, which include losses, provide slightly higher effective permittivity values than, e.g., this method formulated under the assumption of perfect conductors. As expected, the results of both methods tend to converge to the same values for increasing conductor thickness which is demonstrated in Fig. 5(b) for the shielded slotline. Open slotline values are shown for comparison.

The upper curve in Fig. 5(a), however, shows this method to yield higher values for the effective permittivity of the microstrip line than in [17] although the previous investigation would lead to exactly the opposite prediction. It is believed that this problem is caused by two facts. Firstly, the system determinant in [17] is solved for complex eigenvalues while in this paper, only the zeros of the real part are determined. Secondly, a top-cover shielding is used in [17] (distance not specified) whereas in the present method, the structure is considered to be open with sidewall boundary conditions sufficiently far away (at least ten times the strip width).

A similar effect is observed in Fig. 5(c) for the conductor-backed slotline. Following the comparison of Fig. 5(b), the reference values for the effective permittivity [16] should be slightly higher than those calculated by this 


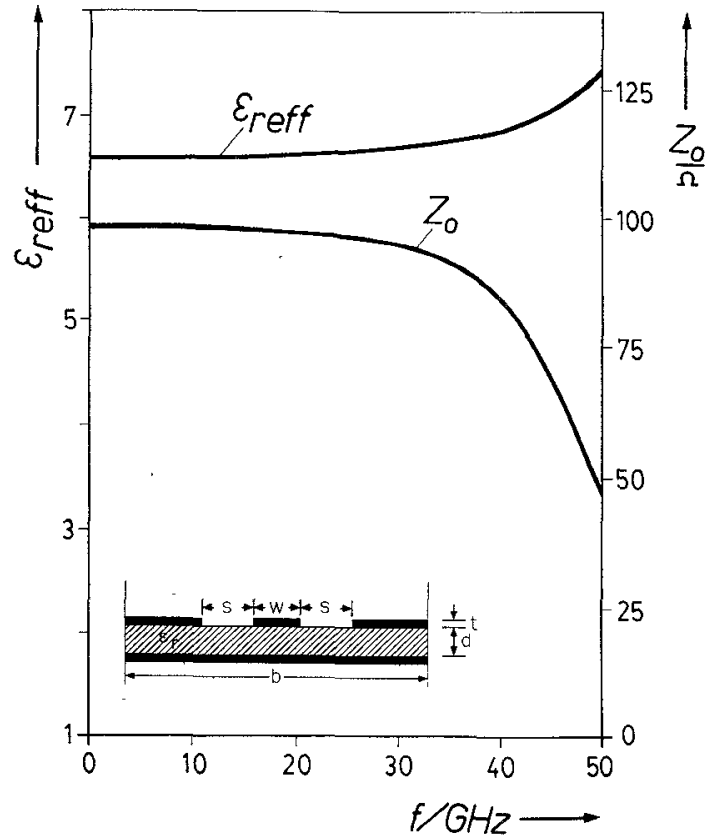

(a)

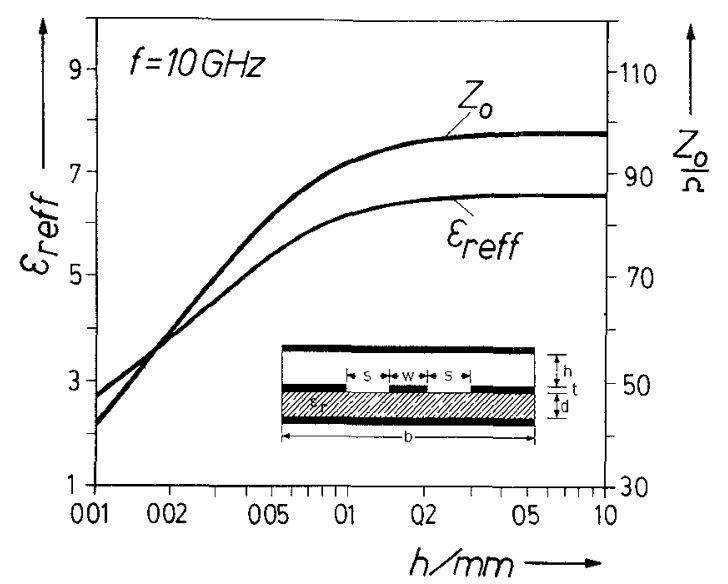

(b)

Fig. 6. Characteristics of conductor-backed MMIC coplanar waveguide and influence of upper-shielding ( $b=1.2 \mathrm{~mm}, d=300 \mu \mathrm{m}, t=$ $5 \mu \mathrm{m}, w=80 \mu \mathrm{m}, \epsilon_{r}=12.9$ ). (a) Effective relative permittivity and characteristic impedance versus frequency. (b) Influence of upper shielding at $10 \mathrm{GHz}$.

method. Surprisingly enough, they are in excellent agreement, even for the higher-order mode. Only for the characteristic impedance, some slight deviations can be observed, which are in a reasonable order of magnitude to be attributed to the difference between lossy and lossless analysis. It should be noted, however, that the scattering-type transverse resonance formulation presented here can be easily implemented on a standard 386 personal computer while the method described in [15]-[17] requires sophisticated computing facilities.

The line characteristics of a conductor-backed coplanar waveguide for MMIC applications are illustrated in Fig. 6(a). The structure can be used up to approximately $30 \mathrm{GHz}$. Beyond this frequency, the electric field concentrates between the center conductor and the ground plane, hence reducing the voltage across the slot and, therefore, the characteristic impedance. Fig. 6(b) shows the influence of an upper shielding for this structure. It is concluded that if $h$ is larger than the substrate thickness $d=300 \mu \mathrm{m}$, the influence of the upper shielding on the line characteristics is only marginal.

\section{Conclusion}

A scattering-type transverse resonance formulation is presented which constitutes a versatile and powerful yet rigorous method for the calculation of transmission-line characteristics of practical MIC, MHMIC and MMIC structures. Different boundary conditions as required for open, conductor-backed or shielded configurations are combined with a general setup for microstrip, slotline or coplanar waveguide. Due to the representation of boundary conditions by reflection coefficient matrices, the transverse resonance algorithm is maintained even though structures and boundary conditions are changed. Compared with other methods requiring mainframe support, the presented formulation provides the advantage of achieving equally accurate results with personal-computer operational software.

\section{REFERENCES}

[1] R. Sorrentino, "Transverse Resonance Technique," in Numerical Techniques for Microwave and Millimeter-Wave Passive Structures, T. Itoh, Ed., New York: Wiley, 1989, ch. 11.

[2] R. Vahldieck and J. Bornemann, "A modified mode-matching technique and its application to a class of quasi-planar transmission lines," IEEE Trans. Microwave Theory Tech., vol. MTT-33, pp. 916-926, Oct. 1985.

[3] J. Bornemann and F. Arndt, "Calculating the characteristic impedance of finlines by transverse resonance method," IEEE Trans. Microwave Theory Tech., vol. MTT-34, pp. 85-92, Jan. 1986.

[4] R. R. Mansour and R. H. MacPhie, "A unified hybrid-mode analysis of planar transmission lines with multilayer isotropic/ anisotropic substrates," IEEE Trans. Microwave Theory Tech., vol. MTT-35, pp. 1382-1391, Dec. 1987.

[5] A. A. Oliner and R. Lampariello, "The dominant mode properties of open groove guide: An improved solution," IEEE Trans. Microwave Theory Tech., vol. MTT-33, pp. 755-763, Sept. 1985.

[6] H. Shigesawa, M. Tsuji, and A. A. Oliner, "Conductor-backed slot line and coplanar waveguide: Dangers and full-wave analyses," in 1988 IEEE MTT-S Intl. Microwave Symp. Dig., pp. 199-202.

[7] R. W. Jackson, "Considerations in the use of coplanar waveguide for millimeter-wave integrated circuits," IEEE Trans. Microwave Theory Tech., vol. MTT-34, pp. 1450-1456, Dec. 1986.

[8] _ "Mode conversion at discontinuities in finite-width conductor-backed coplanar waveguide," IEEE Trans. Microwave Theory Tech., vol. 37, pp. 1582-1589, Oct. 1989.

[9] I. Wolff, D. Kiefer, and S. Bedair, "Considering some undesired effects due to dense packaging in supported coplanar waveguide MMIC's by using combined methods," in 1989 IEEE MTT-S Intl. Microwave Symp. Dig., pp. 657-660.

[10] M. F. Iskander and T. S. Lind, "Electromagnetic coupling of coplanar waveguides and microstrip lines to highly lossy dielectric media," IEEE Trans. Microwave Theory Tech., vol. 37, pp. 1910-1917, Dec. 1989.

[11] T. Rozzi, F. Moglie, A. Morini, E. Marchionna, and M. Politi, "Hybrid modes, substrate leakage and losses of slotline at millimeter-wave frequencies," IEEE Trans, Microwave Theory Tech., vol. 38, pp. 1069-1078, Aug. 1990.

[12] M. Drissi, V. F. Hanna, and J. Citerne, "Analysis of coplanar waveguide radiating end effects using the integral equation technique," IEEE Trans. Microwave Theory Tech, vol. 39, pp. 112-116, Jan. 1991. 
[13] S. S. Bedair and I. Wolff, "Fast and accurate analytic formulas for calculating the parameters of a general broadside-coupled coplanar waveguide for (M)MIC applications," IEEE Trans. Microwave Theory Tech., vol. 37, pp. 843-850, May 1989.

[14] G. Ghione and C. U. Naldi, "Coplanar waveguides for MMIC applications: Effect of upper shielding, conductor backing, finiteextent ground planes, and line-to-line coupling," IEEE Trans. Microwave Theory Tech., vol. MTT-35, pp. 260-267, Mar. 1987.

[15] W. Heinrich, "Full-wave analysis of conductor losses on MMIC transmission lines," in 1989 IEEE MTT-S Intl. Microwave Symp. Dig., pp. 911-914.

[16] _ "The slot line in uniplanar MMIC's: Propagation characteristics and loss analysis," in 1990 IEEE MTT-S Intl. Microwave Symp. Dig., pp. 167-170.

[17] Heinrich, W., "Full-wave analysis of conductor losses on MMIC transmission lines," IEEE Trans. Microwave Theory Tech., vol. 38, pp. $1468-1472$, Oct. 1990.

[18] H. J. Finlay, R. H. Jansen, J. A. Jenkins, and I. G. Eddison, "Accurate characterization and modeling of transmission lines for MMIC's," IEEE Trans. Microwave Theory Tech., vol. 36, pp. 691-697, June 1988.

[19] R. K. Hoffman, J.-P. Kurzweg, and J.-P. Mutzig, "Measurement of the effective permittivity and of the resonator-Q of slot lines on ceramic substrate in the frequency range between $1 \mathrm{GHz}$ and 18 GHz," (in German), Frequenz, vol. 27, pp. 32-40, Feb. 1973.

[20] S. B. Cohn, "Slotline and a dielectric substrate," IEEE Trans. Microwave Theory Tech., vol. MTT-17, pp. 768-778, Oct. 1989

[21] J. B. Knorr and K.-D. Kuchler, "Analysis of coupled slots and coplanar strips on dielectric substrate," IEEE Trans. Microwave Theory Tech., vol. MTT-23, pp. 541-547, July 1975.
[22] S.-T. Peng and A. A. Oliner, "Guidance and leakage properties of a class of open dielectric waveguides: Part I-Mathematical formulations," IEEE Trans. Microwave Theory Tech., vol. MTT-29, pp. 843-855, Sept. 1981.

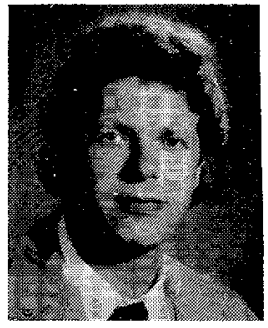

Jens Bornemann (M'87-SM'90) was born in Hamburg, West Germany, on May 26, 1952. He received the Dipl.-Ing. and the Dr.-Ing. degrees, both in electrical engineering, from the University of Bremen, West Germany, in 1980 and 1984, respectively.

From 1980 to 1983 , he was a Research and Teaching Assistant in the Microwave Department at the University of Bremen, working on quasi-planar waveguide configurations and computer-aided E-plane filter design. After a two year period as a consulting engineer, he joined the University of Bremen again, in 1985, where he was employed at the level of Assistant Professor. Since April 1988, he has been an Associate Professor at the University of Victoria, Victoria, BC, Canada. His current research activities include microwave system design and problems of electromagnetic field theory in integrated circuits and radiating structures.

Dr. Bornemann was one of the recipients of the A.F. Bulgin Premium of the Institution of Electronic and Radio Engineers in 1983. He serves on the editorial board of the IEEE Transactions on Microwave THEORY AND TECHNIQUES, and has authored and coauthored more than 50 technical papers. 